\title{
The problem of the second wind turbine - a note on a common but flawed wind power estimation method
}

\author{
F. Gans, L. M. Miller, and A. Kleidon \\ Biospheric Theory and Modeling Group, Max Planck Institute for Biogeochemistry, Hans-Knoell-Strasse 10, \\ 07745 Jena, Germany
}

Correspondence to: F. Gans (fgans@bgc-jena.mpg.de)

Received: 29 April 2010 - Published in Earth Syst. Dynam. Discuss.: 8 June 2010

Revised: 19 March 2012 - Accepted: 11 May 2012 - Published: 8 June 2012

\begin{abstract}
Several recent wind power estimates suggest that this renewable energy resource can meet all of the current and future global energy demand with little impact on the atmosphere. These estimates are calculated using observed wind speeds in combination with specifications of wind turbine size and density to quantify the extractable wind power. However, this approach neglects the effects of momentum extraction by the turbines on the atmospheric flow that would have effects outside the turbine wake. Here we show with a simple momentum balance model of the atmospheric boundary layer that this common methodology to derive wind power potentials requires unrealistically high increases in the generation of kinetic energy by the atmosphere. This increase by an order of magnitude is needed to ensure momentum conservation in the atmospheric boundary layer. In the context of this simple model, we then compare the effect of three different assumptions regarding the boundary conditions at the top of the boundary layer, with prescribed hub height velocity, momentum transport, or kinetic energy transfer into the boundary layer. We then use simulations with an atmospheric general circulation model that explicitly simulate generation of kinetic energy with momentum conservation. These simulations show that the assumption of prescribed momentum import into the atmospheric boundary layer yields the most realistic behavior of the simple model, while the assumption of prescribed hub height velocity can clearly be disregarded. We also show that the assumptions yield similar estimates for extracted wind power when less than $10 \%$ of the kinetic energy flux in the boundary layer is extracted by the turbines. We conclude that the common method significantly overestimates wind power potentials by an order of magnitude in the limit of high wind power ex-
\end{abstract}

traction. Ultimately, environmental constraints set the upper limit on wind power potential at larger scales rather than detailed engineering specifications of wind turbine design and placement.

\section{Introduction}

Several recent studies have quantified large-scale or global wind power availability by extrapolating kinetic energy availability from measured wind speeds (Archer and Jacobson, 2005; Sta. Maria and Jacobson, 2009; Lu et al., 2009; Liu et al., 2008; Leithead, 2007).

Although the exact methodologies may differ, these studies use a similar procedure. Hereafter, we will collectively refer to these studies as the "common method". This methodology can be generally described as follows:

1. Observations of wind velocities, $v$, are derived for a large spatial area from a collection of surface stations (e.g. Archer and Jacobson, 2005), satellite measurements (e.g. Liu et al., 2008), or reanalysis data (e.g. Lu et al., 2009).

2. Technical specifications for a turbine are then used to specify the rotor height, rotor-swept area, and velocity dependent power characteristics.

3. Generated power resulting from the extracted kinetic energy is calculated for a single turbine by $P_{\text {ex }}=\frac{1}{2} \rho C_{\mathrm{f}} A_{\text {rotor }} v^{3}$, where $\rho$ is the air density, $C_{\mathrm{f}}$ is the capacity factor dependent on the selected turbine, and $A_{\text {rotor }}$ is the rotor-swept area. 
4. The area is then populated with wind turbines at a density limited by engineering constraints related to turbine wake turbulence, under the basic assumption that wind turbines do not influence the wind field outside their specific influential wake volume (e.g. Sta. Maria and Jacobson, 2009).

These studies collectively result in high wind power availabilities and exclude effects on larger scale atmospheric flow, since they assume kinetic energy consumption is limited to the turbine wake, but does not affect the large-scale flow.

In stark contrast, studies by Keith et al. (2004) and Wang and Prinn (2010) use global climate models which include consumptive effects and induced feedbacks from large-scale wind power extraction. These two studies also show significant climatic impacts of wind power removal on the climate system. Additionally, Miller et al. (2011) included these consumptive effects and estimated the maximum global landbased wind power availability from the atmospheric boundary layer (ABL) to be $18-68 \mathrm{TW}$, which is much lower than previous estimates and unavoidably associated with significant climatic differences.

The discrepancy in results between the two different methodologies is significant and must be resolved. Advocates of the common method usually argue that imprecisions in general circulation models (GCMs) and the associated representation of wind turbines therein cause the differences in climatic effects, and therefore the results should be disregarded (e.g. see open discussion on Miller et al., 2011). In our opinion the assumption of an unaltered wind velocity outside the turbine wake is the main reason for this difference, because it neglects the effects of turbine-related momentum removal from the atmospheric boundary layer.

The aim of this paper is to investigate the effect of not accounting for kinetic energy and momentum removal from the atmospheric flow during wind power extraction on different magnitudes of power extraction.

We will present a simple model which is based on the balance of momentum and kinetic energy fluxes in the atmospheric boundary layer. We then compare the results of this simple model with the GCM simulation results of Miller et al. (2011).

\section{Momentum and kinetic energy extraction from the atmospheric boundary layer}

Wind turbines generate electrical power by removing kinetic energy from the atmospheric flow. The instantaneous rate of kinetic energy extraction from a moving fluid can be expressed as

$P=F \cdot v$

where $v$ is the velocity of the fluid, and the force $F$ is the rate of momentum extraction from the flow. Wind power is there- fore proportional to the extracted momentum and the velocity at which this momentum is extracted. In general, the highest potential of extracting wind power is realized when there is a high velocity as well as a continuous source of momentum that can be utilized.

Equation (1) forms the basis for deriving the wind power formula used in the common methodology. The momentum density of a fluid moving at a velocity is $\rho v$. The momentum flowing through the cross-section of a single turbine equals the momentum density multiplied by the flow velocity, $J_{p}=\rho v \cdot v$, where $J_{p}$ is the horizontal momentum transport in the fluid. The assumption that the drag from turbines, $F_{\text {turbine }}$, is proportional to $J_{p}$ (i.e. $F_{\text {turbine }} \propto \rho v^{2}$ ) leads to the commonly known wind power extraction equation $P_{\mathrm{ex}} \propto A \rho v^{3}$, where $A$ is the wind-swept area of the wind turbine blades.

This equation is likely to give the impression that wind power availability is determined by measured velocities and air densities only, but its derivation shows that the flux of momentum is equally important. Wind turbines depend on the extraction of momentum, and in the absence of a continual source of momentum, wind turbines would eventually stop the air from moving. In order to quantify the largescale extractability of wind energy, it is therefore essential to quantify and model the sources of momentum and kinetic energy, thereby investigating their influence on wind power extractability.

We next consider the case in which we account for the momentum balance of the fluid from which kinetic energy is extracted. In the limit case of a completely developed wind farm, where all the surface area is equally covered with equidistant wind turbines, lateral sources of momentum can be neglected and only vertical fluxes of momentum add to the overall momentum balance of the system. In the absence of external forces, momentum can not be generated or dissipated within the boundary layer - it can only be transferred from regions with higher velocities to regions with lower velocities. In the atmospheric boundary layer, momentum is transferred from higher altitude layers with higher wind velocities to lower levels with lower wind velocities and eventually to the surface, where $v=0$.

We define two reference heights in the atmospheric boundary layer (see Fig. 1). One is the top of the boundary layer, defined here as $2 \mathrm{~km}$ above the surface. The second layer of interest is the hub-height, which we take to be $80 \mathrm{~m}$ above ground.

\subsection{Fundamental balance equations}

The balance equations for momentum and kinetic energy at the two reference heights are the sum of all vertical fluxes that enter and leave the layer. For simplicity, we assume these balances are in a steady state. 


\subsubsection{Momentum balances at hub-height}

The balance of momentum fluxes at hub-height is written as:

$\frac{\mathrm{d} p_{\text {hub }}}{\mathrm{d} t}=0=F_{\text {transfer }}-F_{\text {surface }}-F_{\text {turbine }}$

where $p_{\text {hub }}$ is the momentum per unit surface area at hubheight, $F_{\text {transfer }}$ is the vertical transport of momentum from above, $F_{\text {surface }}$ is the momentum transferred to the surface by frictional dissipation and $F_{\text {turbine }}$ is the momentum extracted by wind turbines. From this momentum balance, we can derive that an increased momentum extraction by wind turbines, $F_{\text {turbine }}$, must be balanced either by (a) decreased dissipation at the surface, $F_{\text {surface}}$, or (b) increased momentum influx from higher layers, $F_{\text {transfer. In common parame- }}$ terizations of surface momentum transfer, the rate of transfer is a monotonic function of the wind velocity, $v$. This means that we expect a reduction in wind velocities at hub-height with increased values of $F_{\text {turbine, }}$, which in return will reduce extractable wind power associated with this momentum extraction. However, if a reduction in surface dissipation is the main source of momentum for wind turbines, then the extracted wind power is limited by the rate of momentum transfer to the surface, $F_{\text {surface }}$. Under this assumption the kinetic energy dissipation associated with surface momentum transfer gives a first-order estimate of maximum power extraction.

The potential change in momentum transfer from higher altitudes with increased wind turbine extraction cannot be estimated ad hoc. Assuming that the hub-height velocity decreases, one would expect an increase in momentum transfer from above because there is a higher vertical velocity gradient. However, to fully understand this effect, one must know how the velocity reduction at hub-height affects wind velocities in higher layers. To do so, we define another model height at the top of the ABL. The momentum balance equation at the top is:

$\frac{\mathrm{d} p_{\text {top }}}{\mathrm{d} t}=0=F_{\text {acc }}-F_{\text {transfer }}$

where $F_{\text {acc }}$ is the accelerating force or, equivalently, the momentum flux into the top of the atmopsheric boundary layer per unit area.

Note that so far, we based our equations and reasoning only on physical conservation laws. No assumptions have been made about how the fluxes are parameterized. In order to calculate some example fluxes of momentum and kinetic energy and to understand how wind velocities change due to turbine momentum extraction, we will now formulate a model based on parametrizations of momentum fluxes.

\subsubsection{Flux parameterizations}

In the next step, we parametrize the exchange fluxes of momentum between the two model layers. We use standard atmospheric boundary layer theory (Stull, 1988) to formulate these fluxes depending on the hub-height velocity, $v_{\text {hub }}$, and the top-of-ABL velocity, $v_{\text {top. }}$. The transport of momentum between the top-layer and hub-height layer is expressed as:

$F_{\text {transfer }}=\rho C_{\text {transfer }} \cdot\left(v_{\text {top }}-v_{\text {hub }}\right) \cdot v_{\text {top }}$

where $C_{\text {transfer }}$ is a dimensionless momentum transfer coefficient between the two layers which can be derived for a given surface roughness.

The surface friction is parameterized as:

$F_{\text {surface }}=\rho v_{\text {hub }}^{2} C_{\mathrm{DN}}$

where $C_{\mathrm{DN}}$ is the surface drag coefficient for hub-height wind velocities. Assuming neutral stability conditions, the surface drag coefficient for wind speeds at $z=80 \mathrm{~m}$ hubheight can be calculated using

$C_{\mathrm{DN}}=k^{2}\left(\ln \left(\frac{z}{z_{0}}\right)\right)^{-2}=0.0013$

where $k \approx 0.4$ is the von-Kármán parameter and we use a roughness length typical for grasslands, $z_{0}=1 \mathrm{~mm}$ (Stull, 1988).

\subsubsection{Kinetic energy balance}

In addition to the momentum fluxes, we are interested in the vertical flux of kinetic energy. Analogous to the momentum balance, one can formulate balance equations for the vertical fluxes of kinetic energy at each model layer, which is

$\frac{\mathrm{dKE}_{\text {top }}}{\mathrm{d} t}=0=J_{\text {in }}-J_{\text {transfer }}-D_{\text {transfer }}$.

Here, $J_{\text {in }}=F_{\text {acc }} \cdot v_{\text {top }}$ is the kinetic energy imported into the top of the ABL, $J_{\text {transfer }}=F_{\text {transfer }} \cdot v_{\text {hub }}$ is the kinetic energy transferred to hub-height, and $D_{\text {transfer }}$ is the energy lost by turbulent dissipation between the two layers. The kinetic energy balance equation at hub-height is:

$\frac{\mathrm{dKE}_{\text {hub }}}{\mathrm{d} t}=0=J_{\text {transfer }}-D_{\text {surface }}-P_{\text {ex }}$

where $D_{\text {surface }}=F_{\text {surface }} \cdot v_{\text {hub }}$ is the kinetic energy dissipated at the surface and $P_{\mathrm{ex}}$ is the kinetic energy extracted by wind turbines.

\subsection{Turbine parameterization}

For the parametrization of the wind turbines, we use the standard wind power equation and the turbine specifications of the Tjaereborg $2 \mathrm{MW}$ wind turbine with a rotor diameter of $D=61 \mathrm{~m}$ (Sta. Maria and Jacobson, 2009). We assume that the wind farm is very large, so we can neglect energy and momentum influxes from the sides of the wind farm. In this setup each turbine extracts a certain amount of momentum and kinetic energy from the atmosphere, given by the equations:

$F_{\text {ex }}=\frac{1}{2} \rho v_{\text {hub }}^{2} C_{\mathrm{f}} A_{\text {rotor }} / A_{\text {turbine }}$ 
and

$P_{\mathrm{ex}}=\frac{1}{2} \rho v_{\mathrm{hub}}^{3} C_{\mathrm{f}} A_{\text {rotor }} / A_{\text {turbine }}$

where $C_{\mathrm{f}} \approx 0.56$ is the capacity factor, $\rho$ is the air density, $A_{\text {rotor }}$ is the rotor-swept area and $A_{\text {turbine }}$ is the ground surface area that a single wind turbine uses. This parameter describes the turbine spacing in the wind farm.

What is not specified in the model is how $F_{\text {acc }}$ and $J_{\text {in }}$ respond to kinetic energy extraction by wind turbines. In order to derive such a relationship, one would have to model the dynamics of the higher atmosphere, which is not within the scope of this simple model but is investigated later in Sect. 3. Here, we will investigate three different scenarios of how the top of the ABL fluxes might respond to changes in surface momentum extraction: (1) a prescribed hub-height velocity corresponding to the common method, (2) a prescribed momentum flux, $F_{\text {acc }}$, and (3) a fixed kinetic energy flux, $J_{\text {in }}$.

\subsubsection{Assumption (1): prescribed hub-height velocity}

In the first scenario, we assume that the wind turbine installations do not have an effect on the bulk wind velocity, $v_{\text {hub }}$, outside the wake volume, which is equivalent to the assumptions made by the common method. This assumptiom does not necessarily ensure that all kinetic energy and momentum fluxes are balanced. In order to ensure a constant hub-height wind velocity while maintaining the balance of momentum fluxes, the momentum import into the top of the ABL must increase with increased turbine installations to compensate for the increased momentum extraction by wind turbines. This would lead to the following relationship between turbine momentum extraction and total momentum import:

$F_{\mathrm{acc}}=F_{\mathrm{acc}, 0}+F_{\mathrm{ex}}$

where $F_{\text {acc, } 0}$ is the momentum flux without turbine installations and $F_{\text {ex }}$ is the momentum extracted by wind turbines. Using this relationship all the other fluxes and velocities can be determined.

\subsubsection{Assumption (2): prescribed import of momentum}

In a second scenario, we will assume that the momentum input into the top of the ABL is constant and is not affected by wind turbine installations.

$F_{\text {acc }}=F_{\text {acc }, 0}$.

Under this assumption the momentum flux into the hubheight level is fixed. This means that increased wind turbine momentum extraction must be balanced by a decrease in turbulent surface dissipation, which is only possible when the bulk wind velocity decreases. We will therefore expect a lower wind power extractability compared to assumption (1).

\subsubsection{Assumption (3): prescribed import of kinetic energy}

Our third scenario assumes that the kinetic energy flux per unit time is fixed when altering the momentum extraction at the surface. This assumption implies that the momentum flux at the top of the ABL is:

$F_{\text {acc }}=J_{\text {in }, 0} / v_{\text {top }}$

where $J_{\text {in, } 0}$ is the fixed flux of kinetic energy into the boundary layer. Since the total kinetic energy import is fixed, we would expect that power extraction of turbines should saturate with increased turbine densities. Hence, this assumption should also lead to lower estimates of wind power extractability than assumption (1).

\subsection{Results}

In our setup, we assume a hub-height wind velocity of $v_{\text {hub }}=10 \mathrm{~m} \mathrm{~s}^{-1}$ in the absence of wind turbines, which results in an accelerating force of $F_{\text {acc }}=0.168 \mathrm{Ws} \mathrm{m}^{-3}$ and a wind velocity at the top of the ABL of $v_{\text {top }}=12.8 \mathrm{~m} \mathrm{~s}^{-1}$. The kinetic energy fluxes for this scenario in the absence of wind turbines $\left(F_{\text {turbine }}=0\right)$ are: $J_{\text {in }}=2.15 \mathrm{~W} \mathrm{~m}^{-2}$ and $J_{\text {transfer }}=1.68 \mathrm{~W} \mathrm{~m}^{-2}$, which is the total input of kinetic energy into the ABL and hub-height level. The dissipation rates are $D_{\text {transfer }}=0.47 \mathrm{~W} \mathrm{~m}^{-2}$ during the downward transport between top and hub-height, and $D_{\text {surface }}=1.68 \mathrm{~W} \mathrm{~m}^{-2}$ below hub-height.

The fluxes of momentum and kinetic energy and the velocities at the model heights were calculated for different wind turbine densities under the three assumptions. Figure 3 shows the response of the energy and momentum fluxes to changes in wind turbine density. Under assumption (1) the extracted power is a linear function of the number of wind turbines and increases accordingly. The import of kinetic energy and momentum also increase with increasing turbine density so as to offset extraction by the wind turbines, while hub-height velocities outside the wake volume remain constant.

The results of assumptions (2) and (3) resemble each other, both showing a saturating maximum in extracted wind power once a certain density of turbines is reached. Wind velocities decrease with increasing turbine numbers, while kinetic energy and momentum input remain in the same order of magnitude.

For low turbine densities at less than 0.1 turbines per $\mathrm{km}^{2}$, all assumptions lead to very similar results. For higher densities assumption (1) significantly differs from the other two assumptions by predicting much higher values of wind power extractability, kinetic energy and momentum influx from higher altitudes, and constant hub-height velocities at the wind turbine interface. The divergence of the different assumptions is apparent where the turbine power extraction is $\approx 10 \%$ of the total kinetic energy import to the boundary layer. 


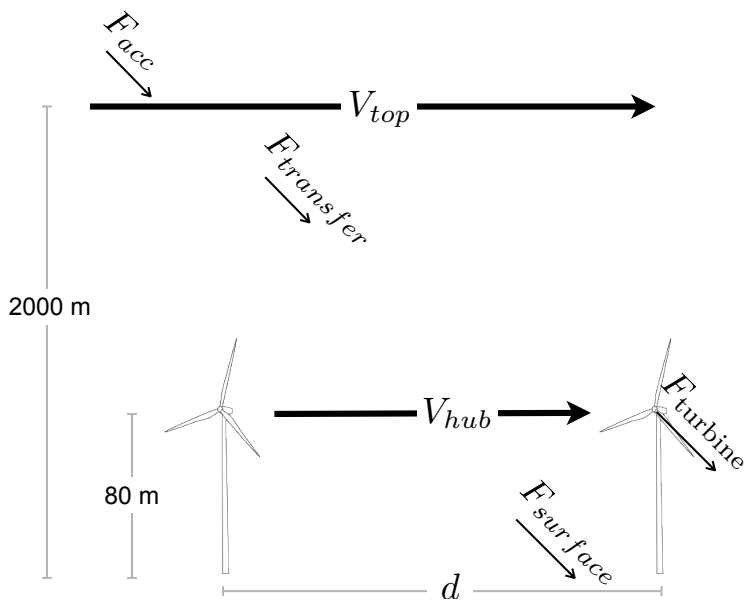

Fig. 1. Schematic illustration of the vertical fluxes of horizontal momentum in the atmospheric boundary layer. Momentum is imported from the upper atmosphere at the rate $F_{\text {acc }}$ and diffuses to lower

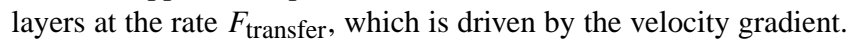
Wind turbines extract some of this momentum at $v_{\text {hub }}$ to generate electrical energy $\left(F_{\text {turbine }}\right)$.

We then compared these estimates for the case of a turbine-specific land area $A_{\text {turbine }}=10 D \cdot 3 D$, where $D$ is the diameter of the wind turbine, as suggested by Sta. Maria and Jacobson (2009). According to assumption (1), the downward momentum flux would need to increase 10 -fold to $0.92 \mathrm{Ws} \mathrm{m}^{-3}$ and the power input into the $\mathrm{ABL}$ would be $17.8 \mathrm{~W} \mathrm{~m}^{-2}$, which is also an increase by roughly an order of magnitude. Using assumptions (2) and (3) leads to relatively low changes in energy and momentum import at the top of the ABL. A comparison of the energy fluxes implied by the different assumptions at this specified turbine density is shown in Fig. 2

The results of assumption (1) pose the question of how the atmospheric circulation would be able to increase power generation by an order of magnitude. Because an explanation of the processes that would lead to such an increase has not been given yet, it seems unlikely that this drastic change is actually possible.

\section{Comparison to climate model simulations}

\subsection{Climate model description}

In order to evaluate which assumption is most reasonable and whether the atmosphere can generate much more power, we need to resort to a GCM that explicitly represents momentum balance constraints by being based on the Navier-Stokes equations of fluid dynamics. We use the climate model simulations of Miller et al. (2011) and compare their results with the ones derived by the 3 different scenarios. We are particularly interested in how the boundary layer kinetic energy import responds to increased momentum extraction at the
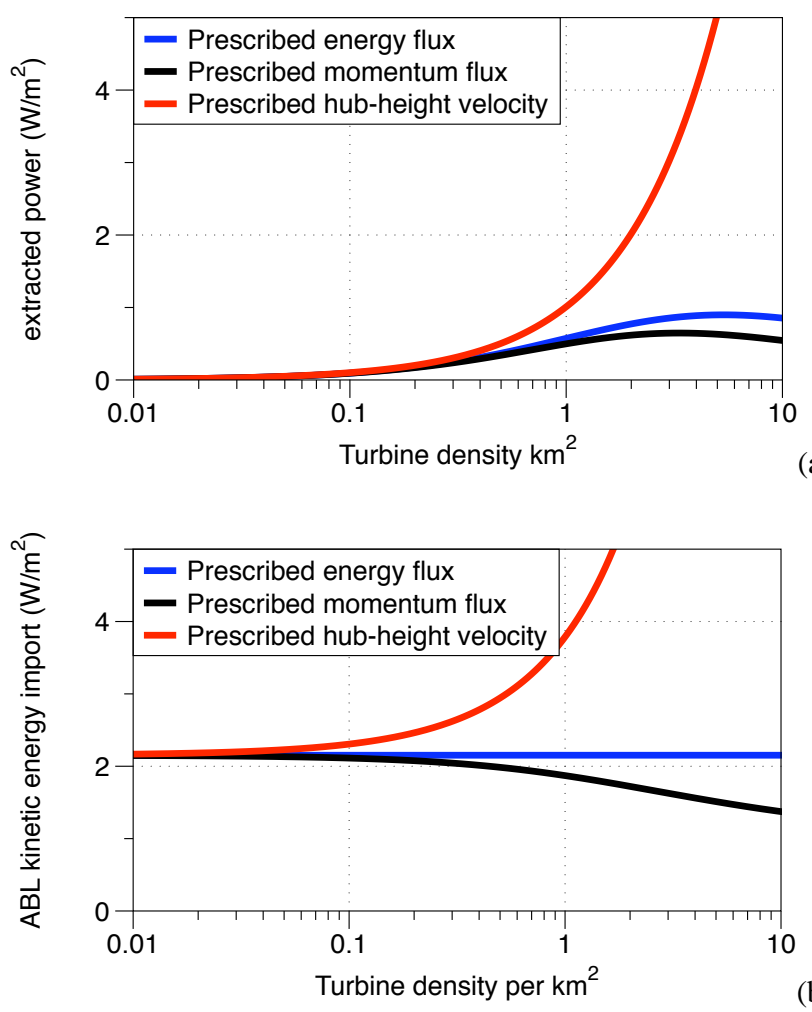

(b)

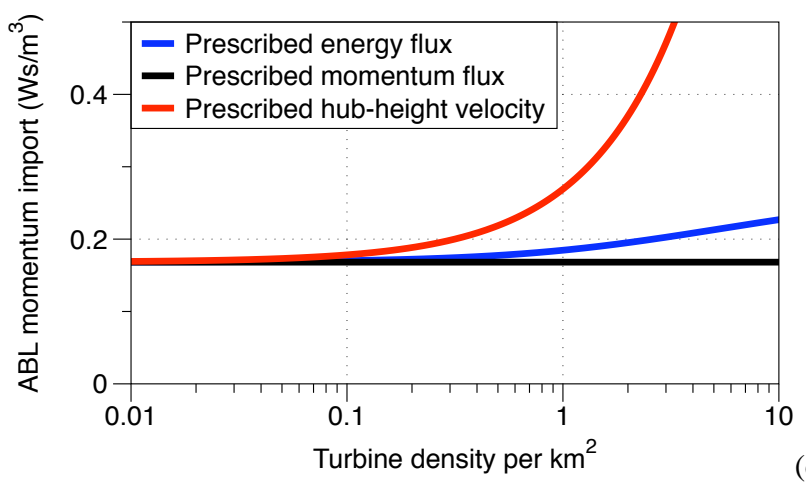

(c)

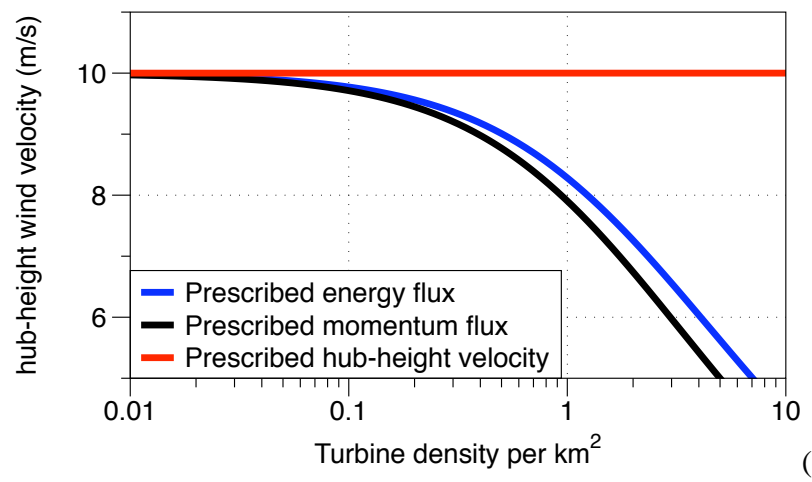

(d)

Fig. 2. Comparison of the simple atmospheric boundary layer model results under 3 different assumptions represented by different colors. The graph shows the turbine power extraction $\left(\mathrm{W} \mathrm{m}^{-2}\right)$, kinetic energy import at the top $\left(\mathrm{W} \mathrm{m}^{-2}\right)$, momentum import at the top $\left(\mathrm{Ws} \mathrm{m}^{-3}\right)$ and wind velocity at hub-height $\left(\mathrm{m} \mathrm{s}^{-1}\right)$. 
Prescribed hub-height velocity

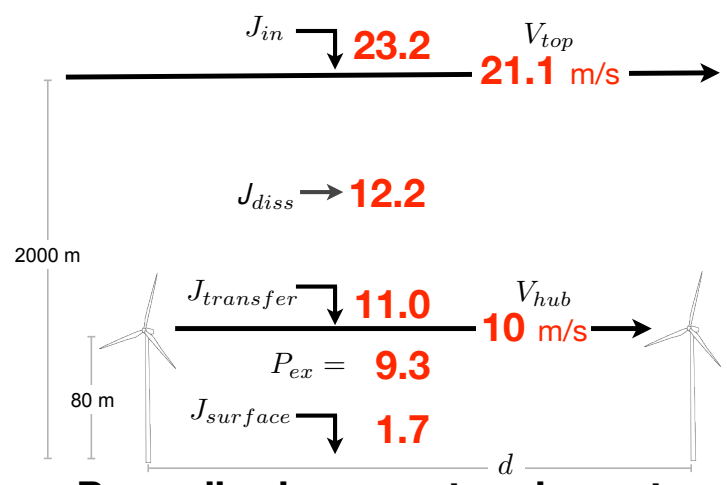

Prescribed momentum import

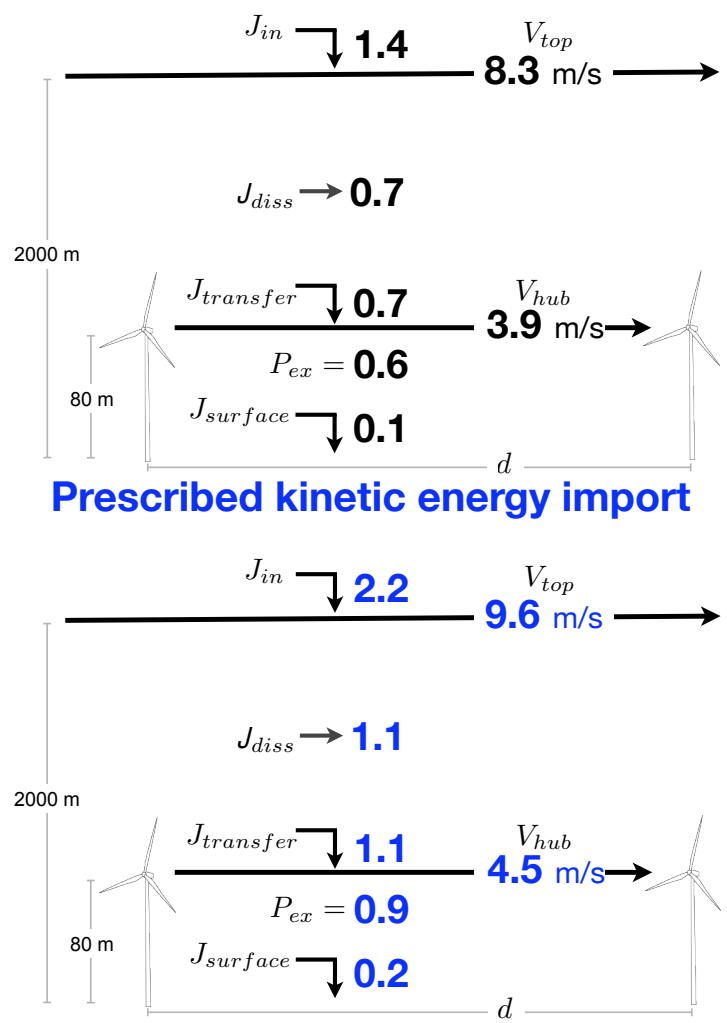

Fig. 3. Comparison of the kinetic energy fluxes for the 3 different assumptions using a turbine spacing of $A_{\text {turbine }}=10 \mathrm{D} \cdot 3 \mathrm{D}$, where $D=61 \mathrm{~m}$ is the rotor diameter. Unless otherwise noted, all quantities have the units $\mathrm{W} \mathrm{m}^{-2}$.

surface. We can therefore determine if the increase in power generation predicted by the common method is supported by GCM results.

In these simulations, wind turbines are represented as an additional frictional force in the atmospheric boundary layer. Details of the study are described in Miller et al. (2011). The wind turbines, represented by the additional frictional parameter $C_{\mathrm{ex}}$, cause a frictional force on the atmospheric boundary layer, $F_{\text {fric }}=\rho C_{\text {ex }} v^{2}$. The conversion efficiency from wind power extracted from the atmosphere to mechanical power of the turbine is estimated to be $\approx 0.6$ using the Betz limit. Extracted wind power per unit area is then

$P_{\mathrm{ex}}=0.6 \cdot F \cdot v=0.6 \rho C_{\mathrm{ex}} v^{3}$.

To represent the common method, wind velocities were taken from a control simulation without any additional friction. Based on these velocities, the power output of a single turbine at each time step was calculated using Eq. (9). The total power extraction per unit surface area is then

$P_{\text {tot }}=\frac{1}{A_{\text {turbine }}} P_{\text {ex }}=\frac{1}{2} \rho \frac{N}{A_{\text {Land }}} C_{\mathrm{f}} A_{\text {rotor }} v^{3}$

where $\frac{1}{A_{\text {turbine }}}$ is the turbine density on land. Comparing Eqs. (14) and (15) lets us derive the relationship between the friction parameter $C_{\mathrm{ex}}$ and the turbine density:

$\frac{1}{A_{\text {turbine }}}=\frac{1.2 C_{\mathrm{ex}}}{C_{\mathrm{f}} A_{\text {rotor }}}$.

For example, a turbine density of 1 turbine per $\mathrm{km}^{2}$ would correspond to a friction parameter $C_{\mathrm{ex}}=0.0013$. The kinetic energy import into the boundary layer of the GCM is diagnosed using the energy balance Eq. (8), resulting in

$J_{\text {in }}=D_{\mathrm{BL}}+P_{\text {ex }}$

where $D_{\mathrm{BL}}$ is the rate of kinetic energy dissipation within the boundary layer and $P_{\mathrm{ex}}$ is the turbine associated power extraction.

\subsection{Results}

The calculated power extraction as well as the influence of wind turbine density on boundary layer wind velocities and boundary layer power input is shown in Fig. 4. For low turbine densities, the common method and the GCM results lead to very similar results. As the turbine density increases, the estimates begin to diverge. During the process of kinetic energy extraction, wind velocities decrease and therefore kinetic energy extractability is reduced. The onset of the divergence takes place when $\approx 10 \%$ of the kinetic energy dissipated in the boundary layer is extracted by the turbines, consistent with the results of the simple model.

The GCM simulations do not show any evidence that the atmosphere would respond to kinetic energy extraction by simply generating more kinetic energy, which is predicted by assumption (1) in our simple model. Figure 4 shows the implied increase in kinetic energy import assuming that kinetic energy is extracted and wind velocities are assumed to be unaffected outside the turbine wake. However, the existence of such a generation mechanism is not supported by the GCM results. Instead, the total boundary layer kinetic energy import decreases in response to kinetic energy extraction and is therefore most similar to the results of assumption (2) with prescribed momentum import. 


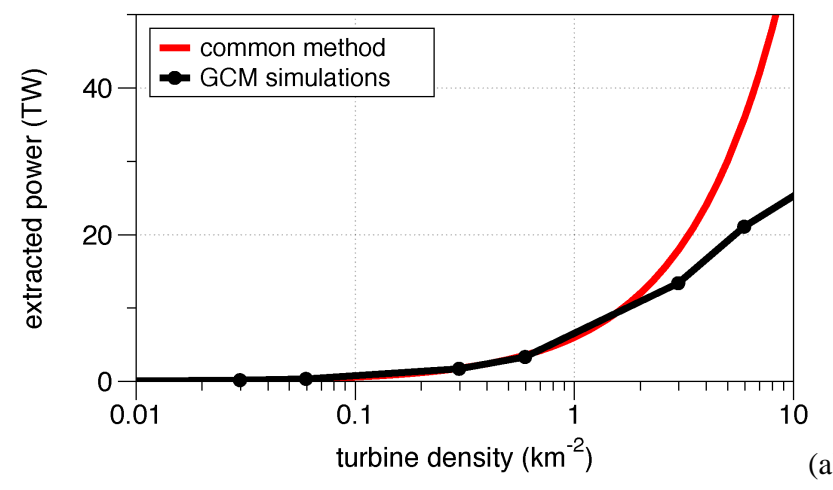

(a)

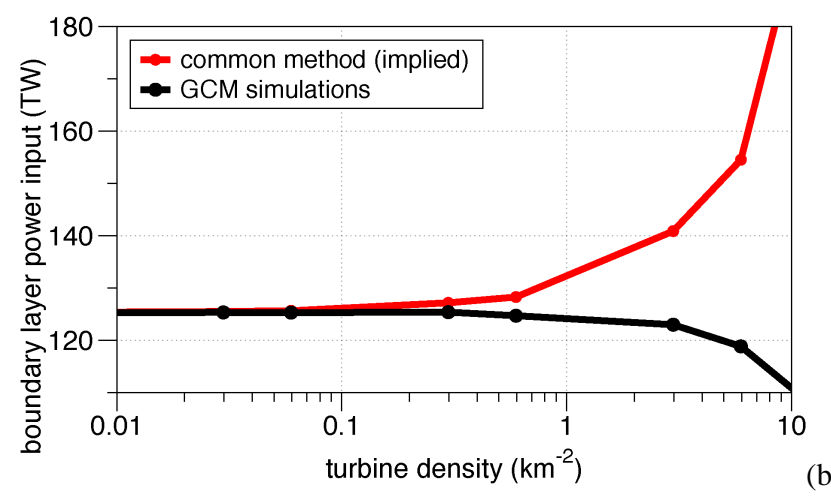

(b)

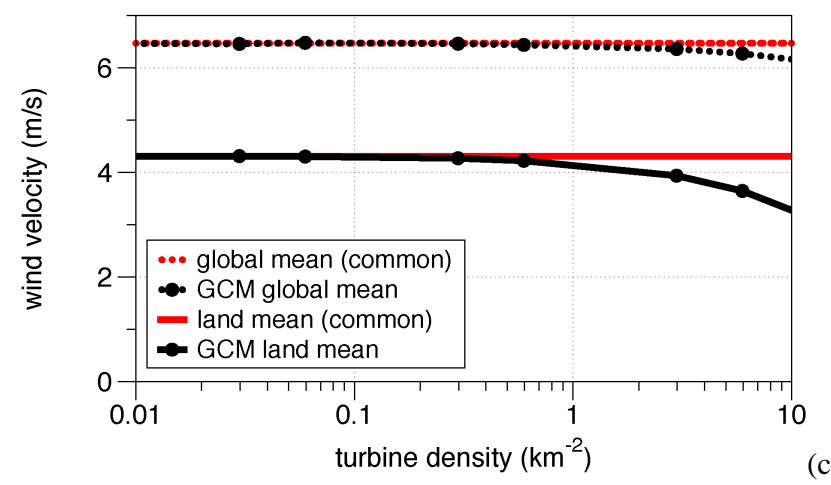

(c)

Fig. 4. Sensitivity of wind power extraction and consequences for boundary layer flow from a set of climate model simulations in which all land regions are populated by wind turbines of different density. Shown are (a) mean power extraction by wind turbines, (b) mean boundary layer kinetic energy import, and (c) mean global wind velocities, and mean land wind velocities. The estimates of the common method are depicted by red lines and as modeled by the GCM are shown as black lines. Note the divergence of the two lines starting at $\approx 2$ turbines per $\mathrm{km}^{2}$, or a power extraction of $10 \%$ of the total kinetic energy import into the boundary layer.

\section{Discussion}

This simple illustration as well as more complex GCM simulations show that accounting for the balance of momentum and kinetic energy is critical to the quantification of large-scale extractability of wind power. Here, we will discuss thermodynamic limits on wind energy generation rates within the atmosphere and link them with predictions made by GCM simulations. We then discuss how these predictions meet the currently observed state of the atmosphere.

Theory: Using well-established energetics of the atmosphere, the global generation rate of kinetic energy is estimated to be approximately 900 TW (Lorenz, 1955; Peixoto and Oort, 1992), about half of which is imported into the boundary layer. Less known thermodynamic derivations show that this rate of kinetic energy generation is at a maximum value given the present-day radiative forcing gradients, as demonstrated by simple theoretical considerations (Lorenz, 1960), box models (Paltridge, 1978; Lorenz et al., 2001) and general circulation model simulations (Kleidon et al., 2003, 2006). This therefore means that an increase in total atmospheric power generation due to wind turbine installations as formulated in assumption (1) should not be expected. Thermodynamic considerations therefore support the use of prescribed kinetic energy import or prescribed momentum import, but rule out the assumption of a prescribed hub-height velocity, because it implies a significant increase in kinetic energy generation.

GCM results: This rather theoretical view is supported by the results of our GCM. In this more complex model, kinetic energy generation does not increase due to additional wind turbine installations. Instead, the kinetic energy flux to the boundary layer decreases in response to very high wind turbine densities (see Fig. 4) and therefore contradicts the assumption of unaffected hub-height wind velocities. Every GCM is based on equations ensuring the conservation of momentum, which is the main reason for the noted divergence in large-scale wind power estimates. So although the specific quantities of extractable wind power and related wind turbine density will certainly depend on specifications of the chosen GCM and its parameterization of wind turbines, the qualitative behavior should be reproducible with different models.

Observations: Advocates of the common method routinely point out that this approach is based on observations, therefore making it superior to the theoretical modeling approaches presented here. In response, we want to stress that the present-day installation scale of wind farms over the global surface is still quite small. The results we have shown here reflect that for these small installation densities, the wind power estimates from the various scenarios are quite similar. Thus, observational evidence actually reinforces none of the scenarios. This fact makes it even more critical then to use a thermodynamically consistent estimation method supported by fundamental physics. That has been completed here, highlighting the resulting error when one applies a linear function derived from small scale wind farm installations to global-scale estimates of wind power potential. Considering the role of atmospheric momentum and kinetic energy transfer in estimating large-scale wind power is essential for reliable estimates on wind power extractability. 


\section{Conclusions}

The ability of wind turbines of any size, quantity, or spatial arrangement to appropriate atmospheric kinetic energy is limited, first by the generation rate of kinetic energy in the atmosphere, and secondly, by the ability of these turbines to appropriate wind power from the atmospheric flow. The common method assumes that the bulk velocity is not affected by the wind power extraction, thereby overestimating available wind power. Taken further, applying this "common method" for large-scale wind power estimates makes the resulting estimate dependent on the turbine characteristics (swept-area, hub height, torque-to-electricity conversion efficiency, spatial arrangement), rather than the generation rate of motion within the atmosphere. While initially counter-intuitive, here we have illustrated why it is the generation rate of kinetic energy in the atmosphere and the associated momentum fluxes, and not a more detailed understanding of wind velocities globally, that ultimately constrains wind power estimates to realizable limits.

It should also be clear that we are presently well below these limits to extraction. In 2008, the wind power production was $0.034 \mathrm{TW}$, or $0.2 \%$ of the 2008 global energy demand of 16.5 TW (Arvizu et al., 2011). If wind farms continue to increase in scale, our results suggest that physical limitations imposed by the atmosphere, estimated here using basic physical principles, will decrease and ultimately limit the power production of successive wind turbines. While it remains unclear how much energy the human population will demand in the future, studying these fundamental limits of renewable energy will ultimately present possible pathways to a renewable future. At a bare minimum though, to ensure that these estimates are relevant and scientifically defensible, careful attention must be paid to ensure that basic physical principles are being obeyed.

Acknowledgement. The authors thank Ryan Pavlick and Nathaniel Virgo for their comments which improved this manuscript.

The reviewers (Valerio Lucarini, Klaus Hasselmann, Daniel KirkDavidoff and two anonymous referees) also aided in clarifying several concepts and conclusions that appear in the final version of this manuscript. This work has been partially supported by the Helmholtz research alliance "Planetary Evolution and Life".

Edited by: H. Held

\section{References}

Arvizu, D., Bruckner, T., Edenhofer, O., Estefen, S., Faaij, A., Fischedick, M., Hiriart, G., Hohmeyer, O., Hollands, K. G. T., Huckerby, J., Kadner, S., Killingtveit, A., Kumar, A., Lewis, A., Lucon, O., Matschoss, P., Maurice, L., Mirza, M., Mitchell, C., Moomaw, W., Moreira, J., Nilsson, L. J., Nyboer, J., PichsMadruga, R., Sathaye, J., Sawin, J., Schaeffer, R., Schei, T.,
Schlömer, S., Seyboth, K., Sims, R., Sinden, G., Sokona, Y., von Stechow, C., Steckel, J., Verbruggen, A., Wiser, R., Yamba, F., and Zwickel, T.: Technical Summary, in: IPCC Special Report on Renewable Energy Sources and Climate Change Mitigation, edited by: Edenhofer, O., Pich-Madruga, R., Sokona, Y., Seyboth, K., Matschoss, P., Kadner, S., Zwickel, T., Eickemeier, P., Hansen, G., Schlömer, S., and von Stechow, C., Cambridge University Press, Cambridge, UK and New York, NY, USA, 2011.

Archer, C. L. and Jacobson, M. Z.: Evaluation of global wind power, J. Geophys. Res., 110, D12110, doi:10.1029/2004JD005462, 2005.

Keith, D., DeCarolis, J., Denkenberger, D., Lenschow, D., Malyshev, S. L., Pacala, S., and Rasch, P.: The influence of large-scale wind power on global climate, P. Natl. Acad. Sci., 101, 1611516120, 2004.

Kleidon, A., Fraedrich, K., Kunz, T., and Lunkeit, F.: The atmospheric circulation and states of maximum entropy production, Geophys. Res. Lett., 30, 2223, doi:10.1029/2003GL018363, 2003.

Kleidon, A., Fraedrich, K., Kirk, E., and Lunkeit, F.: Maximum entropy production and the strength of the boundary layer exchange in an atmospheric general circulation model, Geophys. Res. Lett., 33, L06706, doi:10.1029/2005GL025373, 2006.

Leithead, W.: Wind energy, Philos. T. Roy. Soc. A, 365, 957-970, 2007.

Liu, W. T., Tang, W., and Xie, X.: Wind power distribution over the ocean, Geophys. Res. Lett., 35, L13808, doi:10.1029/2008GL034172, 2008.

Lorenz, E.: Available potential energy and the maintenance of the general circulation, Tellus, 7, 271-281, 1955.

Lorenz, E.: Generation of available potential energy and the intensity of the global circulation, Dynam. Climate, Pergamon Press, Oxford, UK, 86-92, 1960.

Lorenz, R., Lunine, J., Withers, P., and McKay, C.: Titan, Mars, and Earth: entropy production by latitudinal heat transport, Geophys. Res. Lett., 28, 415-418, 2001.

Lu, X., McElroy, M. B., and Kiviluoma, J.: Global potential for wind-generated electricity, P. Natl. Acad. Sci., 106, 10933, doi:10.1073/pnas.0904101106, 2009.

Miller, L. M., Gans, F., and Kleidon, A.: Estimating maximum global land surface wind power extractability and associated climatic consequences, Earth Syst. Dynam., 2, 1-12, doi:10.5194/esd-2-1-2011, 2011.

Paltridge, G. W.: The steady-state format of global climate, Q. J. Roy. Meteorol. Soc., 104, 927-945, 1978.

Peixoto, J. P. and Oort, A. H.: Physics of climate, American Institute of Physics, Springer-Verlag, New York, USA, 1992.

Sta. Maria, M. R. V. and Jacobson, M.: Investigating the effect of large wind farms on energy in the atmosphere, Energies, 2, 816838, 2009.

Stull, R. B.: An introduction to boundary layer meteorology, Kluwer Academic Publishers, Dordrecht, The Netherlands, 1988.

Wang, C. and Prinn, R. G.: Potential climatic impacts and reliability of very large-scale wind farms, Atmos. Chem. Phys., 10, 2053 2061, doi:10.5194/acp-10-2053-2010, 2010. 